\title{
Author Index to Abstracts
}

(Numbers cited refer to abstract numbers)

Adler S, 73

Aiges $\mathrm{H}, 11$

Alagille D, 1, 64

Ala-Houhala M, 87

Alvarez F, 3

Amarri $S, 93$

Ambühl P, 39

Amedee-Manesme O, 29

André C, 75

Andre FE, 68

Arató A, 43

Arato A, 44

Arató A, 31

Arco A, 38

Arjomaa P, 20

Armitstead JCM, 77

Auricchio S, 15, 37, 38, 40, 41

Ballabio A, 15

Ballabriga A, 67

Ball F, 82

Balli F, 55

Barnes N, 14

Barranco F, 67

Bartelsman JW, 79

Baumann W, 82

Belli D, 81

Bender SW, 32

Berg JD, 33

Bernard O, 1, 3, 63, 64

Bertele-Harms RM, 17

Beyreiss H, 26

Beyreiss K, 16

Bhave SA, 4

Bisset WM, 27

Blanc W, 11

Bochner A, 50

Boehm G, 26

Boix-Ochoa J, 67

Bonamico M, 42

Booth IW, 16, 33, 48, 49, 86, 96

Botazzo GJ, 72

Branski D, 73

Brechot C, 63

Brennan EA, 24

Broto J, 67

Brown GA, 16, 96

Broyart JP, 95

Bujanover Y, 93

Buys CHCM, 5

Cadranel S, 35, 53, 68, 80

Calne R, 14

Capano G, 41

Cardelli M, 40

Carlier C, 29

Carrion V, 92

Catassi C, 90

Cezard JP, 95

Cézard JP, 56

Chen M, 23

Clark J, 70

Coe A, 85

Cohen MB, 21

Colombo C, 13

Coppa GV, 90

Cornell HJ, 37

Corney G, 12
Cucchiara S, 51, 55

Cunha Ferreira RMC, 24

Dahlström KA, 71

Dandrifosse G, 18

Danielsson L, 71

Daum F, 11

D'Auria G, 38

Davis L, 58

Delacroix D, 35

Delemos-Chiarandini C, 3

De Luca G, 51

De Prez C, 80

de Ritis $G, 37,38,40$

Descos B, 75

de Sousa JS, 65

De Vincenzi M, 37, 38, 40

Dillon $\mathbf{M}, 7$

Di Lorenzo C, 53, 80

Doherty DG, 12

Dominguez P, 67

Donaldson PT, 12

Duchateau J, 35

Dufour C, 18

Dupont C, 56

Eastham EJ, 30

Edwards MA, 86

Elliott EJ, 24, 76, 77

Eran M, 28, 73

Esposito C, 41

Faber J, 28

Farthing MJG, 24, 76, 77

Fasano A, 78, 83

Felici L, 90

Fenton TR, 7

Ferguson A, 36

Fernandes J, 5

Ferrara A, 78, 83

Forget $P, 18$

Foucaud P, 56

Freier S, 28, 73

Fulconis F, 25

Gabrielli $\mathrm{O}, 90$

Gabrielsson N, 2

Gasparini N, 83

Gaze H, 39

Gellermann B, 10

Gentile V, 41

Giannella RA, 21

Giorgi PL, 90

Glupczynsky Y, 80

Gobio Casali L, 51, 55

Goossens H, 80

Goss-Sampson M, 84

Grandjean CJ, 91

Greco L, 51

Green A, 86

Guandalini S, 78, 83

Guarino A, 21

Hadchouel M, 1, 63, 64

Hadchouel P, 1

Hällström O, 34, 45, 46

Ham H, 53
Harms HK, 17

Heller-Schöch G, 88

Hermier M, 75

Heymans HSA, 79

Hill S, 70, 72

Hjelte L, 2

Hopkinson DA, 12

Houwen RHJ, 5

Howard ER, 66

Hübner C, 10

Hugon RN, 63

Hunt JB, 76

Hunziker W, 15

Iglesias J, 67

Infante $D, 67$

Jakobsson I, 19

Janas M, 87

Jenkins HR, 6, 7

John IR, 4

Jung HYP, 91

Juvonen P, 19

Kahn E, 11

Kalin M, 7

Keller I, 32

Keller KM, 82

Kelly D, 85

Kirchmann H, 32, 82

Klemola T, 31, 43

Knutton S, 69

Kohlschütter A, 10

Koskinen M, 87

Koskinen T, 87

Kuch M, 32

Lachaux A, 75

Latte $F, 40$

Lebenthal E, 22, 92, 93

Lee PC, 22, 92

Lenaerts C, 81

Lentze MJ, 39, 94

Lifschitz $\mathrm{CH}, 23$

Lindberg T, 19, 74

Lindner $S, 10$

Lindquist BL, 22

Lloret J, 67

Lobo-Yeo A, 57, 59, 60

Loeb $\mathrm{H}, 54$

Lothe L, 19, 74

Luzeau R, 29

Magazzù G, 38, 55

Maggiore G, 64

Mahony MJ, 52

Maiuri L, 15, 37, 38, 40

Mäki M, 34, 45, 46, 87

Mäki R, 87

Mancini E, 40

Margarit C, 67

Markowitz J, 11

Martínez Ibáñez V, 67

März C, 17

Mascart-Lemone F, 35

Maurage C, 81

Mazzilli MC, 42
McFarlane IG, 57

McIntosh NI, 7

McNeish AS, 48, 49, 69

McSorley C, 57

Mendes A, 65

Merrick JM, 22

Mieli-Vergani G, 12, 14, 57, 58, $59,60,61,62$

Migliavacca M, 41, 78

Milla PJ, 6, 7, 27, 52, 72, 84

Minoli I, 25

Mirakian R, 72

Morellini M, 42

Morgan MEI, 48, 49

Moro GE, 25

Mouterde O, 56

Mowat AP, 12, 14, 57, 58, 59, 60, $61,62,66$

Müller D, 26

Muller DPR, 84

Murphy MS, 30

Naim HY, 39, 94

Natali PG, 42

Navarto J, 56

Navon $\mathrm{P}, 73$

Nelson R, 30

Newell SJ, 48, 49

Nichols BL, 23

Nicotra MR, 42

Nocerino A, 78

Nützenadel W, 9

Oguchi S, 89

Ohyama S, 89

Pandit AN, 4

Paradis K, 3

Pasquinelli C, 63

Patel PB, 16

Pavone V, 38

Peakman M, 58, 61

Pegat MA, 75

Pelham A, 14

Penninga $D, 5$

Perheentupa J, 20

Petell JK, 93

Pett S, 14

Phillips AD, 70

Piepsz A, 53

Pittschieler K, 93

Porta P, 41

Porteu A, 95

Puntis JWL, 33, 86, 96

Raia V, 37, 38, 40

Räihä N, 26

Räihä NC, 25

Richardson CA, 72

Rivers RPA, 27

Robert J, 75

Rodesch P, 80

Rodrigues C, 65

Rolles K, 14

Romain N, 18

Ronchi M, 13

Roy CC, 81 
Rubino A, 78, 83

Safary A, 68

Salmenperä L, 20

Sander G, 88

Sandomenico ML, 83

Sato M, 84, 89

Savilahti E, 20, 31, 43, 44

Scheffer H, 5

Schmitz-Moormann P, 82

Schnackenberg U, 6

Schöch G, 88

Schwartze E, 9

Scotto J, 63

Scriver CR, 8

Sebastio G, 15

Seidel N, 17

Semenza G, 15

Senaldi G, 60, 62
Senger $H, 26$

Setchell KDR, 13

Shenkin A, 85

Shimizu T, 89

Siimes MA, 20

Silano V, 37, 38, 40

Simell 0,8

Smith DW, 8

Spitz L, 52

Ståhlberg M-R, 46

Staiano A, 51

Sterchi EE, 39, 94

Stern M, 10

Stinson MW, 22

Strandvik B, 2

Suhr A, 9

Sule D, 16, 33, 96

Tainio VM, 44
Tainio V-M, 20, 31, 43

Tanner MS, 4

te Meerman GJ, 5

Tenenhouse HS, 8

Tenore A, 83

Tizard J, 14

Topp H, 88

Tormo R, 67

Troncone R, 36

Vajro P, 1

Van Caillie-Bertrand M, 50

Vandenplas $Y, 47,54$

Vanderhoof JA, 91

Vania A, 42

Vatier J, 56

Veereman G, 50

Verga C, 78

Vergani D, 57, 58, 59, 60, 61, 62
Verkasalo M, 43, 44

Verronen P, 34

Viander M, 46

Visakorpi JK, 34, 45, 46, 87

Walker-Smith JA, 24, 70, 72, 76, 77,85

Watson AJM, 76

Watt JB, 27

Weber AM, 81

Weidenfeld $\mathrm{J}, 73$

Wernicke I, 10

Whitehouse D, 12

Williams R, 14

Wirth S, 82

Yabuta K, 89

Yahav J, 92

Yamashiro $\mathrm{Y}, 89$

Zeghlache S, 68 